\section{Frauen und National- sozialismus}

Sigrid Weigel

\section{»Judasfrauen «}

\section{Sexualbilder im Opfer-Täter- Diskurs über den National- sozialismus}

\section{Zu Helga Schuberts Fallgeschichten*}

In jüngster Zeit ist im feministischen Diskurs eine Debatte entstanden, in der die Opfermythen, die lange Zeit die Frauengeschichtsschreibung - vor allem unter dem Paradigma des "Patriarchats« - dominiert haben, in Frage gestellt werden und endlich auch die Verwicklungen von Frauen in die herrschenden Gewalt- und Herrschaftsstrukturen in den Blick geraten. ${ }^{1}$ Naturgemäß spielt hierbei die Geschichte des $\mathrm{Na}$ tionalsozialismus eine zentrale Rolle. Nachdem die Studien zum Faschismus lange Zeit auf die Weiblichkeitsmythen des NS, die Framen-Opfer und den Widerstand von Frauen konzentriert waren, geht es nun darum, sich mit den Varianten weiblicher Taterschaft zu konfrontieren. Um dieses Anliegen geht es auch in dem Buch »Judasfrauen«, das Helga Schubert 1990 mit dem Untertitel "Zehn Fallgeschichten weiblicher Denunziation im Dritten Reich « publiziert bat, kurz nach der Wende in der DDR.

\section{Das Buch}

Darin erzählt Schubert die Geschichten von zehn Frauen, deren Fälle sie überwiegend aus Gerichtsakten rekonstruiert und, wie sie selbst formuliert, literarisch aufbereitet $(9)^{2}$ hat, wobei die Erzählperspektive wechselt: mehrheitlich (6x) wird in der dritten Person über die jeweilige Denunziantin berichtet, manchmal aber versetzt die Autorin sich in Form eines fiktiven Monologs in die Täterin ( $3 x$ ) und einmal auch in die Perspektive eines Opfers. Schubert nennt ihre Fallgeschichten Parabeln, "Parabeln des Verrats", wobei sich ihr Interesse auf die "Auswirkungen eines totalitären Staates auf das Alltagsverhalten seiner Bürger « richtet (7). Die Fälle politischer Denunziation aus der Nazizeit sind für sie damit Beispiele, die zugleich auf ihre eigene Gegenwart in der DDR verweisen. In der wenige Tage nach dem 9. November' 89 geschriebenen Vorbemerkung kann sie dies schon deutlich aussprechen und ihre Geschichten »verschliusselte Botschaften « nennen. Schubert geht also einer bestimmten, gleichsam mittelbaren Täterschaft nach, dem Verrat, genauer der "Versuchung zum Verrat $[. .$.$] in einer$ Gesellschaftsordnung, in der es möglich [ist], private Konflikte sozusagen mittels Staatsgewalt zu lösen « (20). Und diese spezifische Form der Täterschaft, in der die Hände der Täter rein bleiben und sie sich der Staatsgewalt bedienen, um die Opfer zu treffen, wird für sie in den Fällen weiblicher Denunziation verkörpert: in den »Judasfrauen«. Somit hat der Verrat für sie ein weibliches Gesicht: "Judas als Frau« (24).

Die Eingrenzung ihres Vorhabens auf Fälle weiblicher Denumzianten gehört zur Vorentscheidung der Autorin über ihren Gegenstand und bestimmt die Auswahl ihres Materials. Insofern ist der Titel ihres Buches Programm. Damit überkreuzt sich ihr aktuelles, aus der DDR-Erfahrung gespeistes Interesse am Verrat mit dem Interesse an einem ihrer Meinung nach spezifisch weiblichen Tattypus, an einer Schuld, 
die sie besonders den Frauen im NS-Faschismus zuschreibt.

\section{Das diskursive Umfeld}

Ihr Buch hat damit Anteil an jener Literatur, mit der die dichotomischen Positionen von männlichen Tätern und weiblichen Opfern, die den Geschlechtern - in Umkehrung der Hexen- und Megärenbilder männlicher Phantasieproduktionen - von Frauen gerne zugewiesen wurden, keine Geltung mehr haben. Nachdem in $\operatorname{der} \mathrm{Ge}$ genwartsliteratur lange Zeit - am deutlichsten in der "Väter-Literatur « der 70er Jahre $^{3}$ - die Väter als Täter identifiziert wurden. eine Auseinandersetzung mit der Vergangenheit also weitgehend einer Demontage der Vater-Autorität gleichkam, gibt es in jüngster Zeit auch manche Bemühungen, die Mütter aus ihrer Nische historischer Unschuld zu vertreiben, so etwa in Helga Novaks »Die Eisheiligen « (1979) oder in Birgit Pauschs Erzählungen "Die Verweigerungen der Johanna Glauflügel« (1977) und »Das Bildnis der Jakobina Völker « (1980), aber auch schon in Bernward Vespers "Die Reise « (1977). Und es gibt - auch wenn im Genre autobiographischer Texte die Stimmen von Opfern zahlenmäßig weitaus dominieren - vereinzelte Reflexionen, in denen die eigene Verstrikkung erinnert wird, wie etwa in Margarete Hannsmanns $»$ Der helle Tag bricht an. Ein Kind wird Nazi« (1982) oder auch in Christa Wolfs "Kindheitsmuster« (1976). Das kann dann allerdings auch zu einer Wiederholung der damaligen Faszination für die Ideologie der nationalen Erziehungsaufgabe wie zu einer nachträglichen Verharmlosung und Legitimation des eigenen Handelns geraten, wie z.B. in Melita Maschmanns "Fazit. Mein Weg in die Hitler-Jugend" (1979), ${ }^{4}$ das ausgerechnet in der Form eines fiktiven Briefes an eine juidische Freundin daherkommt. ${ }^{5}$ Während sich die Deutschen also immer noch schwertun, sich in ihrer Erinnerung an den NS-Faschismus mit ihrem Ort in dieser
Geschichte, d.h. mit der Perspektive der Täter bzw. der Nachkommen einer Tätergeneration zu konfrontieren, gehört das Schuldtrauma von Überlebenden zum festen Bestandteil autobiographischer Erinnerungen von Juden, die der Shoa im Exil oder sogar im Lager entkommen sind. Das schwer entwirrbare Geflecht von Opfer- und Täterempfindungen - und das mangelnde Interesse ihrer deutschen Umgebung an ihren Erfahrungen - hat beispielsweise das lange Verstummen der Jüdin Grete Weil motiviert, die erst 1980 in ihrem autobiographischen Text »Meine Schwester Antigone« eine Sprache für die Thematisierung ihres Traumas einer unterlassenen Widerstandshandlung fand. Und in der DDR waren Geschichten vom Widerstand und vom "antifaschistischen Kampf « ohnehin mehr gefragt als Erinnerungen an einen Schuldzusammenhang, wobei der Antifaschismus gleichsam die Rolle einer Deckerinnerung an den darin verdrängten Faschismus spielte. ${ }^{6}$

Die in der historischen Forschung und in der Frauenforschung zum Thema »Frauen und Faschismus« lange Zeit vorherrschende Fixierung auf die nationalsozialistische Mutterideologie, die Geschlechterpolitik der Nazis und die Spurensuche nach einem Widerstand von Frauen war z.T. motiviert durch die verbreitete Legende, mit der die Frauen für Hitler verantwortlich gemacht wurden, hatte also vor allem eine Funktion für die Demontage des von links bis rechts, von Jürgen Kuczynski bis Joachim Fest, verbreiteten $M y$ thos »Frauen baben Hitler an die Macht gebracht «, der einer Überprüfung selbst am Beispiel des tatsächlichen geschlechtsspezifischen Wahlverhaltens nicht standhält ${ }^{7}$ - womit die Frage, warum Frauen überhaupt Hitler gewählt und zugejubelt haben, sich natürlich nicht erübrigt. Ebensowenig wie eine Befragung der Verwicklung von Frauen in eben diese Ideologieund Gewaltzusammenhänge überflüssig würde durch Erkenntnisse über den $\mathrm{Zu}$ sammenhang von Antisemitismus und Misogynie oder durch die Studien über den 
männerbündischen Charakter faschistischer Ideologie, wie sie z.B. mit Klaus Theweleits »Männerphantasien « (1977) vorliegen. In ihrer Studie über "Weiblichkeitsmythen« (1983) hat Christine Wittrock beispielsweise Kontinuitäten zwischen dem Frauenbild der vorfaschistischen Frauenbewegung, besonders ihrem konservativen Flügel, und Weiblichkeitsideologien während der Nazizeit untersucht, nur daß sie - unter einem, Anfang der 80er Jahre noch weitgehend unangefochtenen »Emanzipations $\ll$-Postulat "ein durchaus fortschrittliches Frauenbild" bei einem von ihr als oppositionell bewerteten Teil der Nationalsozialistinnen entdeckt (188), ${ }^{8}$ anstatt aufgrund der untersuchten Kontinuität die Ursprünge und Funktionen dieses Fortschrittsbegriffs selbst in Frage zu stellen. Dieser orientiert sich nämlich überwiegend an einer Gleichberechtigung im Sinne gleicher, aktiver und verantwortlicher Teilhabe von Fraven am jeweils bestehenden politischen System, deren Verwirklichung - wenn man sie einmal antizipieren wollte - dann allerdings die Untersuchung geschlechtsspezifischer Verwicklungen in historische Schuldzusammenhänge tatsächlich erübrigte, weil sich beide Geschlechter alle Verantwortung teilten. Tatsächlich aber haben Frauen, auch in untergeordneten Positionen, dort, wo sie z.B. Befehle ausgeführt, wo sie etwa die Bevölkerungs- und Rassenhygienepolitik der Nazis als Fürsorgerinnen in die Praxis umgesetzt haben, oder auch dort, wo sie als Blockwartsfrauen zum Klima gegenseitiger Kontrolle und Gesinnungsfahndung beigetragen haben, den NS-Faschismus aktiv mitgetragen. Besonders in kritischer Auseinandersetzung mit Margarete Mitscherlichs These von der »Friedfertigen Frau« (1987) und in einer Kritik am Mythos von der Unschuld des weiblichen Geschlechts, wie er in Teilen der Frauenbewegung, speziell im Diskurs über »weibliche Utopien«, zum Ausdruck kommt, fragen in letzter Zeit verschiedene Wissenschaftlerinnen ${ }^{9}$ nach dem spezifischen Anteil von Frauen am Natio- nalsozialismus. ${ }^{10}$ Doch konkrete historische Untersuchungen zu dieser Art Frauenbiographien, wie sie beispielhaft in dem von Angelika Ebbinghaus herausgegebenen Buch über "Opfer und Täterinnen« (1987) vorliegen, stecken noch in den Anfängen. Schon diese Studie konfrontiert uns allerdings mit der Tatsache, daß es gerade die sozialfürsorgerischen, die sogenannten helfenden Berufe waren, in denen Frauen in einem erschreckenden AusmaB schuldig geworden sind. Während aber die letztgenannten Debatten und Studien bislang nur in einer kleinen Öffentlichkeit bekannt sind, haben die beiden genannten Mythen nichts an ihrer Popularität verloren; in der Entgegensetzung beider Mythen schreibt sich die Tradition gespaltener Frauenbilder fort, die im Nationalsozialismus selbst - im Bild der reinen, arischen Frau einerseits und in den Zerrbildern der minderwertigen juidischen oder erbkranken Frau andererseits - ihre verheerende Wirkung entfalteten.

\section{Das Programm der »Judasfrauen«}

Helga Schubert steht also mit inrem Vorhaben nicht allein. Sie widmet sich aber im Zusammenhang des genannten Diskurses einem speziellen Thema, der Denunziation durch Frauen, und zwar sehr schwerwiegender Fälle von Denunziation, die oft den Tod des Opfers zur Folge hatten.

Ihre zehn Geschichten berichten von einer Frau, deren Ehrgeiz, Karl Goerdeler wiederzuerkennen, der gesucht war im Kontext der Fahndung nach dem HitlerAttentat vom Juli '44, zu dessen Verhaftung beitrug (1), von einer Schwangeren, Mitglied der NSDAP, die ihren Arzt, selbst Mitglied der NSDAP, wegen sogenannter Flüsterpropaganda anzeigte (2), von einer Blockwartsfrau, die ein Gespräch in der Bahn, von dem sie nur gehört hatte, denunzierte, weil ein Mann sich darin negativ über die Regierung und die Zustände 
geäuBert hatte (3), von einer Frau, die nach mehreren Anzeigen schlieblich als »Vertrauensperson der Gestapo « tätig wurde und die für die Verhaftung mehrerer Männer verantwortlich war (4), von einer Ehefrau, die ihren Mann wegen Anti-Hitler-Äußerungen denunzierte (5), von dem bereits aus der Presse bekannten Fall des Pianisten Karlrobert Kreiten, der von drei Frauen, eine davon Freundin seiner Mutter, angezeigt wurde, weil er Hitler als krank bezeichnet hatte (6), von der Frau eines Lehrers, die eine Gesprächsrunde in einer hessischen Kleinstadt, an der sie selbst teilgenommen hatte, verriet (7), von dem Ehemann einer inhaftierten Jüdin, der durch eine Nachbarin, die sein Vertrauen gewonnen hatte, denunziert wurde (8), von einer Tochter, die nichts dagegen unternahm, als ihre Mutter kurz vor Kriegsende zwei Soldaten dazu aufforderte, sie vor ihrem Mann zu schützen, und diese ihn umbrachten (9), und von dem Gespräch der Autorin mit einer ehemaligen Mitarbeiterin des »Sicherheitsdienstes« im »Dritten Reich« (10).

Mit Ausnahme der letzten Fallgeschichte, die von einer direkten Begegnung mit einer ehemals sehr überzeugten und aktiven Nationalsozialistin berichtet und die in der Schuld-Zuschreibung im Vergleich mit den anderen Fällen merkwürdig unentschieden bleibt, und mit Ausnahme des Schicksals Karlrobert Kreitens hat Helga Schubert ihre Fälle sämtlich Gerichtsakten entnommen: und zwar die Geschichte der Schwangeren einer Originalakte von Freislers Volksgerichtshof aus der Nazizeit, während die anderen sieben Fälle auf Verfahren westdeutscher Gerichte nach '45 zurückgehen, deren Urteile in der 22bändigen Sammlung "Justiz und NSVerbrechen« veröffentlicht sind, die an der Universität Amsterdam erarbeitet wurde. Die Sammlung enthält über 600 Urteile aus Anklagen wegen NS-Tötungsverbrechen, das ist eine Auswahl aus den (bis Ende 1965) gegen mehr als 12.000 Personen ergangenen Urteilen, die in der Sammlung nach zwölf Deliktgruppen - eine da- von ist Denunziation - unterschieden werden. ${ }^{11}$

Die Behauptung, daB »die Mehrzahl aller Denunzianten [...] weiblichen Geschlechts« sei, wie auf dem Waschzettel von Schuberts im Luchterhand Literaturverlag veröffentlichten Buch verkündet ist, wird im Text selbst nicht wiederholt. Sie liebe sich auch aus den Quellen, die Schubert benutzt hat, nicht begründen. Denn selbst wenn die in der 22bändigen Sammlung veröffentlichten Urteile gegen Denunzianten vorwiegend gegen Frauen ergangen sein sollten, wäre noch zu überprüfen, ob dieses Bild bei der Auswahl der 600 aus 12.000 Urteilen - zufällig oder auch nicht - zustandegekommen ist. Und darüberhinaus stellt sich natürlich die weiterreichende Frage, ob wegen Denunziation angeklagte Männer und Frauen vor Gericht gleich behandelt wurden oder ob sich in der Urteilsfindung womöglich ein ähnliches Vorurteil bemerkbar machte, wie es in Schuberts Text zum Ausdruck kommt, daB nämlich Denunziation ein typisch weibliches Verbrechen sei. Durch das ganze Buch nämlich, durch den Titel und die Auswahl der Fälle, wird diese These suggeriert.

Neben der expliziten Auswahl von Fällen weiblicher Denunzianten wird dieses Bild noch durch eine andere, implizite, vielleicht sogar unbewußte Auswahl gestuitzt. Von den zehn Geschichten handeln nämlich neun von Fällen, in denen die Opfer Männer sind, während nur in einer Geschichte sowohl Männer als auch Frauen der Denunziation zum Opfer fielen, wobei die Frauen als Nebenfiguren allerdings keine Konturen gewinnen. So entwirft die Autorin das Bild einer starren Geschlechterdramaturgie, in der sich jeweils ein weiblicher Denunziant, eine "Judasfrau « eben, und ein oder mehrere männliche Opfer gegenüberstehen. Auch in der Geschichte über die Denunziation des Ehemanns einer jüdischen Frau wird die vorausgegangene Denunziation dieser Jüdin durch einen Untermieter zwar kurz erwähnt, ohne daß dieser männliche De- 
nunziant im Text allerdings einer näheren Betrachtung unterzogen wird. Denn das Augenmerk der Autorin gilt den Porträts ihrer Denunziantinnen und deren Opfern. Dies ist eine möglicherweise unbewuBte Konzeption - die Auswahl von Fällen mit fast ausschlieBlich männlichen Opfern wird jedenfalls an keiner Stelle thematisiert -, die so auch zu einer Ausblendung weiblicher Opfer von Denunziation führt. Sie verstärkt das Bild genuin weiblicher Täterschaft bzw. Schuld, das hier entworfen wird.

Dies mag das Unbehagen erklären, das ich nach der Lektiire der Fallgeschichten empfand, auch den Eindruck, eigentlich nichts erfahren zu haben über die Schuldzusammenhänge, in denen Menschen im "Dritten Reich " dazu kamen, ihre Nachbarn oder Verwandten einem Terrorregime auszuliefern, auch nichts über die spezifische Verwicklung von Frauen in das NSSystem. Weil mir aber die Fragestellung der Autorin in dem oben skizzierten Kontext so wichtig erscheint, bin ich diesem Unbehagen nachgegangen, wobei mir einige Verfahrensweisen in Schuberts Darstellung deutlich geworden sind.

\section{Die Isolierung einer Taterin aus einem Schuldzusammenhang}

Die Denunziation Goerdelers z.B. geschah offensichtlich aus einer Gruppensituation heraus, in der die Frau, die Schubert als Denunziantin vorstellt und die sie nur mit einem Vornamen, Helene, nennt - während sie die anderen Beteiligten mit ihrer Funktion und das Opfer beim Namen nennt - sich offenbar nicht getraut hatte, Goerdeler, den sie unter den Gästen identifiziert hatte, zu melden, bis es schließlich nach einem Gespräch unter den in der Gaststube Versammelten einer ihrer Kollegen und ihr Vorgesetzter unternahmen, den Gesuchten zu stellen und auszuliefern. Vermutlich weil Helene bei ihrer Zeugenaussage bewirkte, daß nicht die beiden Kollegen, die sich das "Verdienst « an der
Ergreifung Goerdelers zugeschrieben hatten, sondern sie selbst vom Führet die Belohnung von einer Million überreicht bekam - ohne daB sie allerdings mit dem Geld irgendetwas anzufangen wuBte, denn weder verbrauchte sie es, noch nutzte sie es zur Flucht - wurde sie als Verantwortliche dieser Denunziation nach Kriegsende zunächst zu fünfzehn Jahren und 1947 in der Revision dann zu sechs Jahren Zuchthaus verurteilt, während das Verfahren gegen die beiden beteiligten Männer eingestellt wurde. In ihrer Darstellung nun folgt Schubert dieser gerichtlichen Isolierung einer Täterin aus einem Kollektiv, ohne sich näher mit der für NS-Taten sehr signifikanten Konstellation zu beschäftigen: wie nämlich in einer Gruppe eine Atmosphäre von Übereinstimmung geschaffen wird, in der die Beteiligten ihre Hemmungen gegenüber dem Verrat zurückdrängen, wie also ein Gruppenzusammenhang sich in einen Schuldzusammenhang verwandelt, dem ein einzelner geopfert wird.

Aus mehreren der Geschichten nämlich wird, trotz der Darstellung, die die eine Denunziantin herausstellt und schuldig spricht, deutlich, daB diese jeweils in einem Gesprächszusammenhang mit anderen stand. Da wird über anstößige ÄuBerungen gesprochen, werden diese weitererzählt, bis ein gegenseitiger Druck, die Angst oder die Erwartung entsteht, Meldung zu erstatten. Da werden Vertraulichkeiten mit der Blockwartsfrau oder anderen Funktionsträgern des Regimes ausgetauscht, in denen verbotene oder mißliebige Äußerungen anderer weitergetragen werden, bis schlieBlich einer oder eine die Anzeige übernimmt, vielleicht um sich hervorzutun, vielleicht um sich zu beruhigen, vielleicht aus Unterwerfung oder aus »Übereinstimmungssucht « (Wolf). Wie aber funktionieren solche Ketten oder Netze von Gerede und Informationsaustausch? Welcher "Mikrophysik der Macht« (Foucault), von Angst und Vorteil folgen sie? Wie sind die sozialen Beziehungen hier organisiert, daB in ihnen die ethische Barriere fällt, einen anderen aus- 
zuliefern? Wie kann es geschehen, daB das Opfer soweit entindividualisiert wird, $\mathrm{daB}$ es als Objekt der Bestrafung den Gewaltinstanzen übergehen wird? Wie wirken verändertes (Un)RechtsbewuBtsein, völkische Ideologie und die Affekte des einzelnen hier zusammen? Und wenn es um die Frage der Geschlechterdifferenz geht: Gibt es Unterschiede in der Art und Weise, wie Frauen und Männer in dieser sozialen Ordnung situiert sind und wie sie sich darin verhalten?

All dies wären Fragen für eine literarische Erinnerungsarbeit, die zurückblickt in eine Zeit kollektiver Verstrickung und die zugleich interessiert ist an der, womöglich verborgeneren Gegenwärtigkeit derartiger, in ihrer Gewalt vielleicht veränderter Schuldzusammenhänge. Das Buch von Schubert liefert einmal mehr einen traurigen Beweis dafür, daß eine solche Erinnerungsarbeit nur möglich ist, wenn man sich selbst verwickeln läBt, wenn der Faschismus nicht als vergangenes und nicht als Problem der anderen abgehandelt wird. Mit der Isolierung der einen Täterin aus einem Schuldzusammenhang aber und mit dem Genre dokumentarischer Fallgeschichten, die mit dem Verweis auf die juridischen Quellen auch Autbentizität und Wahrheit für sich beanspruchen, distanziert die Autorin auch die negativen Heldinnen ihrer Geschichten von ihrem eigenen Lebenszusammenhang, hält sie sie sich vom Leibe. Möglicherweise aber sind gerade juridische Quellen am wenigsten geeignet, um den genannten Fragen nachzuforschen.

\section{Die Reproduktion des juristischen Diskurses}

Die Isolierung eines Tàters oder einer Tàterin ist nämlich Effekt einer Darstellung, die sich an Gerichts-Quellen orientiert und damit der Logik juristischer Wirklichkeitskonstitution folgt. Die Behandlung und Wahrnehmung der Nazi-Geschichte vor Gericht erfordert ja die Kleinarbeitung von
Schuldzusammenhängen zu einzelnen Delikten von anklagefähigen und venurteilbaren Tatern, ein Verfahren, wie es nun auch wieder nach dem Ende der DDR favorisiert wird.

Dabei setzt das Täterkonzept vor Gericht immer ein bewuBtes, intentionales und zielgerichtetes Handeln eines einzelnen voraus. ${ }^{12}$ Alle Aspekte der Tat und des Täters, die dies einschränken, fübren zur Minderung der Verantwortung, der Schuld- und Straffähigkeit. Gerade in den Strafprozessen gegen NS-Täter wurde von der Möglichkeit einer Eingrenzung der Eigenverantwortung der Angeklagten - mit dem Argument, es habe kein »eigener Täterwillen « vorgelegen, kein »Interesse am Taterfolg " oder keine wexzessive Tathetei-

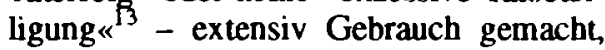
und zwar aus dem Motiv, Entschuldigungs- und Entlastungsgründe für die Angeklagten zu finden, um eine zahlenmäBig nicht zu große Gruppe »Verantwortlicher" aus der Masse des deutschen Volkes isolieren zu können, dessen moralischer Entlastung als ganzes die Verurteilung der einzelnen gleichzeitig zugute kommt.

Im Unterschied zu diesem institutionalisierten Typus deutscher »Vergangenheitsbewältigung " ginge es in der literarischen Erinnerungsarbeit aber gerade darum, die Taten dieser einzelnen in jene Zusammenhänge zu stellen, die sich als Möglichkeitsbedingungen für ihr Schuldigwerden darstellen, bzw. das sichtbare Hineingehen oder auch das unbewuBte Hineingeraten in Schuldzusammenhänge zu rekonstruieren. Dem Diskurs der Urteilsfindung folgend, verurteilt aber Schubert in ihren Fallgeschichten ihre negativen Heldinnen noch einmal, anstatt Fragen nach den Möglichkeitsbedingungen für die Tat zu stellen.

\section{Die Isolierung der Täterin aus dem Gewaltzusammenhang}

Die Orientierung ihrer Darstellung an den Gerichtsquellen hat auch zur Folge, daß sie die Funktion der Gerichte im National- 
sozialismus selbst nicht mit in ihre Befragung einbezieht. Da sie es bei ihren Quellen mit zweierlei Gerichtsverfahren zu tun hat, mit dem Unteil westdeutscher Nachkriegsgerichte, die Verfahren vor Nazi-Gerichten zum Gegenstand hatten, in denen die damaligen Zeugen der Anklage nun Angeklagte sind und die damaligen Angeklagten - sofern sie noch am Leben sind - heutige Zeugen, bewegt sie sich in relativ hermetischen Wirklichkeitsausschnitten, die die ganze Maschinerie des Terrorregimes, in der die Denunziationen ihre grauenvolle Wirkung zeitigten, ausblenden - insbesondere aber die Justiz selbst, denn die Gerichte nach ' 45 zeigten wenig Interesse daran, sich mit der Schuld der Gerichte vor' 45 zu befassen.

In ihrem Einleitungskapitel mit der Überschrift "Judasfrauen « hatte Schubert noch geschrieben:

\section{Von Frauen verraten.}

Von Männern verhaftet, von Männern verhört, von Männern verurteilt, von Männern geköpft.

Aber von Frauen verraten.

Ein leiser Verrat.

Ein heimlicher und sauberer Verrat. Kein Blut an den zarten Handen, das Blut klebt am Fallbeil.

Frauen, die andere Menschen durch ihren Verrat töteten.

Was waren das für Frauen? (16)

Abgesehen von den sprachlichen Klischees - wan den zarten Händen « - wird hier eine bemerkenswerte geschlechtsspezifische Schuldteilung angesprochen, die aber in der Beschreibung der Fallgeschichten nicht weiter verfolgt wird. Die Verleugnung männlicher Schuld geht in Schuberts Text dabei soweit, daB sie ein Gericht als hilflose Instanz darstellt, das durch eine Denunziantin gleichsam dazu gezwungen wurde, ein Todesurteil auszusprechen: »Hilde war darüber so empört, daß sie aufsprang und die Aussagen gegen ihren Mann beschwor. Nun muBte ihn das Kriegsgericht zum Tode verurteilen (91)«.
Nur in einem Fall, den Schubert direkt den Akten des Volksgerichtshofes entnommen hat, wird die Tötungsmaschine NS-Gericht explizit thematisien (50).

\section{Die moralische Verurteilung der Denunziantin}

Da sich Schuberts Darstellung an den Urteilstex ten orientiert, bleibt ihr für das, was sie "literarische Aufbereitung « nennt, nur das Feld einer eigenen Präsentation und Deutung der Fälle. Hierbei konzentriert sie sich auf eine psychologische Interpretation ihrer Denunziantinnen, auf Charakterstudien sozusagen. Damit folgt sie einer durchaus problematischen Tendenz der modernen, durch soziologische und psychologische Ursachenforschung beeinfluBten Justiz, in der nicht mehr die Tat, sondern der/die Täter/in mit der ganzen Biographie und Persönlichkeit vor Gericht steht. Doch während dies im Gerichtsverfahren meistens zur Einschränkung der Schuldfähigkeit verwendet wird und oft zu einem milderen Urteil führt, ist die Psychologisierung bei Schubert Teil ihrer moralischen Be- bzw. Verurteilung der Denunziantinnen. Dabei entwirft sie das Bild von Frauen, die aus überwiegend niederen Motiven handeln: aus Geltungssucht, um sich eigene Vorteile zu verschaffen oder um sich zu rächen oder ihren Mann loszuwerden. Und in ihrem Interesse am moralischen Urteil weicht sie dann schon mal vom Gerichts-Urteil ab, wenn sie sich beispielsweise für die beiden vom Gericht verurteilten Soldaten, die einen Mann erschossen haben, nicht weiter interessiert, dessen Frau und Tochter aber, die vom $\mathrm{Ge}$ richt mangels Beweisen freigesprochen wurden, als die eigentlich Schuldigen darstellt, die die Soldaten aufgefordert hätten, sie von ihrem Mann bzw. Vater zu befreien (163).

Mit ihren Charakterstudien moralisch verwerflicher Frauen wird das Problem der Denunziation aber, anstatt da $B$ man damit tatsächlich konfrontiert würde, gleich- 
sam ad acta gelegt. Denn die böse Frau ist ja immer die andere Frau, mit der weder die Autorin noch die Leser/innen etwas zu tun haben brauchen. Auf diese Weise wird auch die Beunruhigung, die von der historischen Tatsache massenhafter Denunziation im Nationalsozialismus und von der Verstrickung von Frauen in diese Gewaltherrschaft ausgeht, stillgestellt. »Jedesmal grübelte ich wie vor einem Rätsel und war erst erleichtert, wenn ich die Lösung gefunden hatte, « heißt es in der Vorbemerkung (9). Die Lösung: das ist hier wie vor Gericht das Urteil, hier allerdings das moralische Urteil, der Entwurf eines bestimmten Charakter- und Täterinnentyps. Diese "Lösung « bringt Erleichterung, weil sie die andere im Bild fixient und von $\mathrm{Au}-$ torin und Leser/in trennt.

Daran ändert auch die Tatsache nichts, daß einige Fälle aus der Perspektive der Denunziantinnen, als Monolog, beschrieben werden. Vielmehr wird damit eine zusätzliche, den juridischen Quellen nicht mögliche Psychologisierung vorgenommen, da die Autorin ihren Heldinnen Motive und Empfindungen in den Mund legen kann, vor deren Aufklärung die Gerichte häufig kapitulieren müssen, weil sie sich am Übergang zwischen positiver und subjektiver Wahrheit bewegen. Diese Zone der Unsicherheit und Unklarheit wird in der »literarischen Aufbereitung « Schuberts in Eindeutigkeit überführt. In dem Fall der Lehrersfrau, die ' 54 wegen der Denunziation einer Gesprächsrunde angeklagt wurde, geschieht das z.B. durch einen signifikanten Wechsel zwischen Konjunktiv und Indikativ. Der Fall ist in Form einer fiktiven Rede der Angeklagten an den Richter gestaltet. Während die referierten Zeugenaussagen aus dem Urteil im Konjunktiv gehalten sind:

\section{Der Gelehrte und der Kunstmaler hätten} sich nach der Verhaftung in den Verhören gegenseitig belastet, und zwar so, daß es für beide zum Todesurteil vor dem Volksgerichtshof gereicht hat (133).

Und immer hätte ich, wie es ein richtiger
Lockspitzel tun müsse, provoziert, sagt er, so daß $[. .$.$] (130),$

sind die Motive der Angeklagten für die Autorin dagegen ganz klar:

Ich fühlte mich zurückgesetzt und ausgeschlossen, war neugierig und in meinem Stolz verletzt - ich wollte auch gern dazugehören (122).

Solche Motivstudien schreiben das Denken in Schuldkategorien, das der juristische Diskurs vorgibt, fest und verankem es in einem moralisch untermauerten Opfer-Täter-Schema, das im Hin und Her zwischen Schuldzuschreibung und der Entlastung bzw. Entschuldigung keinen Raum läßt für Imitationen und die Wahrnehmung komplexerer Verstrickungen. "Es steht mir nicht $\mathrm{zu}$, die beschriebenen Frauen zu verurteilen. Heute glaube ich, daB auch sie Opfer der Diktatur waren « (9). Diese ÄuBerung Schuberts in der Vorbemerkung, die exakt das Schema von Verurteilung und Entlastung wiederholt, dabei aber nichts an den Urteilen in der Interpretation der Einzelfälle ändern kann und zudem - indem sie die Täter als Opfer sieht - das Opfer-Täter-Muster wiederholt und verstärkt, dient allein der moralischen Entlastung der Verurteilenden, die, indem sie die Schuldigen zu Opfern macht und kurz darauf auch von ihrem Mitleid spricht, wiedenum ihre eigenen Urteile, ihr eigenes Schuldigwerden an den Objekten ihrer Darstellung entschuldigt. Möglicherweise kommt in diesem Satz ein unbewuBtes Wissen der Autorin darüber zum Ausdruck, da $B$ auch ihr eigener Text denunziatorische Züge trägt.

\section{Die Sexualisierung der Denunziation}

In vielen von Schuberts Fallgeschichten, besonders dort, wo die Denunziation eines Ehemanns durch seine Frau berichtet wird, wie auch in jenen Fällen, die aus der Perspektive der Denunziantin erzählt werden, 
ist die psychologisierende Darstellung mit einer abwertenden Sexualisierung verbunden. Schon das Bild der Denunziantinnen, das in der Vorbemerkung entworfen wird "Sie konnten der Versuchung zum Verrat nicht widerstehen « (9). - konnotiert den Verrat deutlich mit negativen Sexualbildern.

In den Einzelporträts wird das dann durch Weiblichkeitsbilder konkretisiert, in denen die Geltungssucht beispielsweise mit einem sexuellen Begehren unterlegt wird. Am deutlichsten ist das in dem Monolog der Frau, die gleich mehrere Männer anzeigt, wobei ihr jeweils sexuelle Motive unterstellt werden. Als Nymphomanin ist sie auch im Verrat unersättlich, so daB der Verrat gleichsam als eine verschobene Vereinigung mit dem »unerreichbaren Mann « - so der Titel der Fallgeschichte - erscheint. Auch der Lehrersfrau, die wegen der Denunziation einer Gesprächsrunde angeklagt war, wird erotischer Geltungsdrang unterstellt, und es wird das Geriucht wiedergegeben, sie habe zu einem Freund ihres Mannes intime Beziehungen unterhalten. Und die Frau, die angeklagt war, ihren Nachbarn denunziert zu haben, erscheint in sexueller Hinsicht als sehr freizügig und ist zudem noch lesbisch, während von der Frau, die ihren Mann denunzierte, berichtet wird, sie habe einen »liederlichen Lebenswandel« geführt, durchreisende Soldaten mit nach Hause genommen und mit ihnen geschlafen (87). Am verwerflichsten aber erscheint wohl die Tochter, die mit dem Soldaten, der als Mörder ihres Vaters bezeichnet wird, schläft: »Noch in dieser Nacht schlief sie mit ihm. Ich hörte die beiden in dieser Nacht, wie sie schrien vor Lust, ohne Rücksicht auf mich, " heißt es aus der fiktiven Perspektive der unbeteiligten zweiten Tochter (161).

Mit diesem Leitmotiv rundet sich das Bild der moralisch verwerflichen Denunziantinnen ab und paBt sich ohne Rest in die Stereotypen der sexuell unersättlichen, todbringenden und männermordenden Weiblichkeitsmythen ein.
Wenn dieses Frauenbild nun den Namen der "Judasfrau « erhält, dann werden damit auch Verbindungen zwischen Antisemitismus und Misogynie evoziert. Denn auch im rassistischen Antisemtismus trägt das Bild des Juden sowohl Züge des Verrats als auch Züge einer entweder dämonischen oder »weibischen « Sexualität. Insofern könnte man vielleicht auch Schuberts Formulierung »Judas als Frau« - und nicht: die Frau als Judas - als Versprecher lesen, in dem etwas von jenem strukturell rassistischen Deutungsmuster zum Ausdruck kommt, in dem der Verrat als ein genuin weibliches Verbrechen erscheint, das mit der Natur und der Sexualität von Frauen in Verbindung gebracht wird.

\section{Das Vorhaben der Befragung weiblicher Taterschaft bleibt Desiderat}

Das so wichtige Vorhaben Schuberts, die aktive Teilhabe von Frauen an einem Terrorregime zu thematisieren, mündet damit leider im bekannten Mythos der Megäre und in antifeministischen Stereotypen. Damit hat sich in ihrem Text vor allem jener Affekt durchgesetzt, der bereits im Vorwort anklingt:

Mich stört die Frauenveredelung: So sensibel, so zart, so kooperativ, so mütterlich, so mitleidig, so kreativ, so authentisch sind wir nicht. Wir sind auch böse und auch gefährlich, auf unsere Weise. Sobald ein Mensch auf einem Sockel steht, möchte ich den Sockel erschlagen (17). ${ }^{14}$

Der so wichtigen und nützlichen Aufgabe, den Sockel zu zerschlagen, hat Schubert allerdings einen Bärendienst erwiesen, indem sie in ihrem Kampf gegen den Mythos von der unschuldigen Frau nur wieder den Mythos von der qua Geschlecht verwerflichen Frau reproduziert hat.

Dieser Zirkel könnte wohl nur aufgebrochen werden, wenn man in der Erinnerung an Schuldzusammenhänge, wie sie im NS-Faschismus zum deutschen Alltag 
gehörten, das Denken in juristischen oder moralischen Kategorien, vor allem die Isolierung und Fixierung von Tätem und Schuldigen aufgäbe, um den Weg zu einer ganz anderen Art von Wahrnehmung und Verantwortung zu öffnen.

Und was die Frage der Geschlechterdifferenz betrifft, so blieb diese in Schuberts Buch ja - wegen der starren Geschlechterdramaturgie, die der Darstellung vorausgesetzt ist, - eigentlich unbefragt. Unbefragt bleiben die verschiedenen Positionen und möglicherweise unterschiedlichen Verhaltensweisen der Geschlechter in Schuld- und Gewaltzusammenhängen, unbefragt geschlechtsspezifische Formen von Macht, Gewalt und Widerstand. Beispielsweise könnte man die - noch zu überprüfende - Hypothese, daB Frauen eher durch Denunziation als durch unmittelbare Gewalt zum Funktionieren des NSFaschismus beigetragen haben, in Verbindung mit anderen historischen Studien diskutieren, die etwas zur Kulturgeschichte geschlechtsspezifischer Delinquenz und Gewalt beitragen: etwa mit der Beobachtung, daß bei Frauen Verbaldelikte häufiger sind als andere, weil offene Gewalt für das weibliche Geschlecht kulturell tabuisiert war und schwerer geahndet wurde. ${ }^{15}$

Wesentlicher als diese Spur ist aber jedenfalls im Zusammenhang eines Denkens nach dem durch Auschwitz markierten Zivilisationsbruch ${ }^{16}$ - die Erinnerung und Befragung der realen Positionen von Frauen in dieser Geschichte, um sich von lieb gewordenen »Unschuldsritualen " ${ }^{17}$, von bequemen Weiblichkeitsmustern wie auch von generalisierenden Geschichtsbildern wie dem des »Patriarchats « zu trennen.

* Der Beitrag ist die gekürzte Fassung eines Aufsatzes, der demnächst erscheint in Sigrid Weigel: Bilder des kulturellen Gedächnisses. Beitrdge zur Gegerwartsliteratur. DuilmenHiddingsel 1992.

\section{Anmerkungen}

1 Zuleta Lerke Gravenhorst; Carmen Tatschmurat (Hrsg.) (1990): TochterFragen. NS-Frauengeschichte. Freiburg.

2 Die Seitenzahlen beziehen sich auf die Ausgabe Frankfurt/M. 1990.

3 Zur Nach-68er Figur der literarischen Auseinandersetzung mit dem Nationalsozialismus s. Klaus Briegleb/Signid Weigel (Hrsg.) (1992): Sozialgeschichte der deutschen Literatur. Bd. 12: Gegenwartsliteratur nach 1968. München.

4 Erstveröffentlichung 1963 mit dem Untertitel "Kein Rechrfertigungsversuch* (sic!).

5 Vgl. Elisabeth Brainin; Marieta Zeug (1990): Arisch ist der Zopf - Jüdisch ist der Bubikopf. In: Nationalsozialismus und Nationalismus. Materialienband 8 der Frankfurter Frauenschule. Frankfurt/M., S. 7-30.

6 Vgl. z. B. Gertraud Gutzmann (1991): Zum Stellenwert des Spanischen Bürgerkriegs in Anna Seghers' Romanen »Die Entscheidung" und $» D a s$ Vertrauen . In: Inge Stephan/Sigrid Weigel/Kerstin Wilhelms (Hrsg.): "Wen kilmmen's, wer spricht*. Zur Literatur und Kulturgeschichte von Frauen aus Ost und West. Köln, S. $195-210$.

$7 \mathrm{Vgl}$. Annemarie Tröger (1977): Die DolchstoBlegende der Linken: «Frauen haben Hitler an die Macht gebracht $\lll$. In: Frauen und Wissenschaft. Beitrdge zur Berliner Sommenuniversität für Frawen. Juli 1976, Berlin, S. 324-355.

8 Christine Wittrock (1983): Weiblichkeitsmythen. Das Frauenbild im Faschismus und seine Vorldufer in der Frauenbewegung der 20er Jahre. Frankfurt/M.

9 S. besonders Karin Windhaus-Walser (1988): Gnade der weiblichen Geburt? In: Feministische Studien . 6. Jg., H. 1. Vgl. auch das Themenheft "Weimarer Republik und Faschismus. Eine feministische Auseinandersetzung." Die Philosophin 2. Jg., H. 3/1991.

10 Problematisch wird diese Frage nach der Spezifik weiblicher Täterschaft uberall dort, wo aus einer Psychologie »weiblicher Logik« bestimmte weibliche Handlungsmuster abgeleitet werden und damit ein Modell weiblicher Kriminalität entsteht.

11 Amsterdam 1968, Registerband fehlt leider noch immer.

12 Das ist erst mit dem Delikt der »Zugehörigkeit zu einer kriminellen Vereinigung « im Kontext der "Terrorismusbekämpfung" partiell aufgehoben worden, in der der einzelne für die Delikte der gesamten Gruppe haftbar gemacht wird.

13 Adalbert Rückerl (1984): NS-Verbrechen vor 
Gericht. Versuch einer Vergangenheitsbewditigung. Heidelberg, S. $275 \mathrm{ff}$.

14 Hier wird auch die unausgesprochene Spur itres Affektes gegen Christa Wolf spurbar.

15 So das Ergebnis einer Fallstudie von Susanne Burghartz (1990): Leib, Ehre und Gut. Delinquenz in Zuirich Ende des 14.Jahrhunderts. Zirich.

16 Dan Diner (Hrsg.) (1988): Zivilisationsbruch Denken nach Auschwitz. Frankfurt/M.

17 Meike Baader (1991): Unschuldsrituale in der Frauenforschung zum Nationalsozialismus. In: Babylon. Beitrage zur jüdischen Gegenwan. 4. Jg., H. 9, S. 140-145 (Besprechung von Gravenhorst Tatschmurat, vgl. Anm. 1).
Dagmar Reese

\section{Neuere Literatur zum Thema Frauen und National- sozialismus. Ein Besprechungs- essay}

Beilmann, Christel (1989): Eine katholische Jugend in Gottes und dem Dritten Reich. Briefe, Berichte, Gedrucktes 19301945, Kommentare 1988/89 (Reihe Hambach). Wuppertal: Peter Hammer Verlag. 384 S., DM 29,80.

Czarnowski, Gabriele (1991): Das kontrollierte Paar. Ehe- und Sexualpolitik im Nationalsozialismus (Ergebnisse der Frauenforschung, Bd. 24). Weinheim: Deutscher Studien Verlag. 320 S., DM 45,-.

Kinz, Gabriele (1990): Der Bund Deutscher Mädel. Ein Beitrag zur außerschulischen Mädchenerziehung im Nationalsozialismus. Frankfurt a.M/Bern/New York/Paris: Peter Lang. 312 S., DM 78,-.

Sachse, Carola (1990): Siemens, der Nationalsozialismus und die moderne Familie. Eine Untersuchung zur sozialen Rationalisierung in Deutschland im 20. Jahrhundert. Hamburg: Rasch und Röhring. 329 S., DM 58,-.

Siegel, Tilla (1989): Leistung und Lohn in der nationalsozialistischen »Ordnung der Arbeit «. Opladen: Westdeutscher Verlag. 325 S., DM 46,-. 